\title{
Geometric Morphometrics Analysis of Chelae and Carapace of the Freshwater Prawn Macrobrachium Bate, 1868
}

\author{
RICH GEMILANG SIMANJUNTAK ${ }^{1}$, RURY EPRILURAHMAN ${ }^{1 *}$ \\ ${ }^{1}$ Laboratory of Animal Systematics, Faculty of Biology, Universitas Gadjah Mada \\ Jl. Teknika Selatan, Sekip Utara, Yogyakarta, Indonesia. 55281 \\ *Email: rurybiougm@ugm.ac.id
}

Received 11 April 2019; Received in revised form 19 April 2019;

Accepted 23 June 2019; Available online 30 June 2019

\begin{abstract}
The shape of chelae and carapace can be used to distinguish between species of prawn. This study aims to determine the variations in the shape of chelae and carapace in several species belonging to the genus Macrobrachium using analysis of geometric morphometric. This study uses photos of specimens that have been processed with several TPS software. Data analyzed statistically by PCA using the MorphoJ software. Clustering analysis using UPGMA method using PAST software. The results showed the carapace shape grid deformation varied at the tip of the rostrum, the tip of the ocular spine and the lower curvature of the front of the carapace, and the base spines of rostrum. Grid deformation in the shape of chelae varies at the tip of the pollex, the junction between the pollex and the manus on the inferior margin of the propodus, the upper and lower points marking the junction of the dactylus with the propodus. PCA shows the total variation of the carapace shape is $82.66 \%$ which is divided into PC1: $75.11 \%$ and PC2: $7.55 \%$. While the total variation of the shape of chelae is $87.56 \%$ which is divided into PC1: $55.49 \%$ and PC2: $32.07 \%$. Clustering analysis shows the grouping of populations of Macrobrachium, the first group is M. latidactylus and $M$. sintangense, the second group includes $M$. horstii and $M$. latimanus. M. lar is a species that shows the similarity of the shape of the carapace and chelae with the two groups. M. rosenbergii and M. pilimanus are on different lines.
\end{abstract}

Keywords: carapace; chelae; geometric morphometric; Macrobrachium; shape

\section{INTRODUCTION}

Crustaceans show various body shapes and are divided into three parts, namely cephalon, thorax, and abdomen. In general, the first two parts are combined as a cephalothorax. Morphological characters have long been used to study diversity in the genus Macrobrachium. The second pleura in the widening abdomen covers the pleura 1 and 3 , this form is the character of the Caridea group (New, 2002). The shape of chelae and carapace are morphological characters that can be used as characteristics that are typical of each species including prawn. Both rostrum and a pair of second periopods are the main morphological features used for taxonomic identification of species in the genus Macrobrachium (Valencia and Campos, 2007). Indonesia has a large diversity of prawn species of the genus Macrobrachium, seven prawn species of the genus Macrobrachium in Indonesia including Macrobrachium latimanus, Macrobrachium pilimanus, Macrobrachium latidactylus,
Macrobrachium rosenbergii, Macrobrachium horstii, Macrobrachium sintangense, and Macrobrachium lar.

Morphological variations related to reproductive strategies have been extensively studied in Decapoda, especially in the morphology of chelae and carapace in the cephalothorax. One study to see the variation in shape on Macrobrachium is through the geometry morphometrics method. Geometric morphometrics is a new methodology for studying variations of shape through landmark configurations. Geometric morphometrics uses landmarks to visualize shapes (Adam et al., 2004; Rufino et al., 2009). Landmarks are also called anatomical points. This method produces shape variables that can be used to provide visual means and describe patterns of different shapes in the data (Adams et al., 2013; Klingenberg, 2013). Geometric morphometrics is known to be better than traditional morphometric because it is based on the 
analysis of drawing shapes in cartesian coordinates.

\section{MATERIALS AND METHODS}

The tools used in this study are Canon 1200D cameras for shooting the prawn, mini studios as shooting sites, black cloth, rulers, and laptops equipped with some software for analysis including, tpsUtil, tpsDig2, tpsRewl, tpsSuper, tpsSplin, MorphoJ, PAST, and Notepad. The samples used in this study were seven types of Macrobrachium, namely Macrobrachium latimanus, Macrobrachium pilimanus, Macrobrachium latidactylus, Macrobrachium rosenbergii, Macrobrachium horstii, Macrobrachium sintangense, and Macrobrachium lar which were collections from the Laboratory of Animal Systematic, Faculty of Biology UGM.

The study was conducted in 8 months, from April to December 2018. This study used parts of the chelae and carapace of seven types of Macrobrachium. Taking photos using a Canon $1200 \mathrm{D}$ camera is done on the left chelae and carapace facing to the left. Prawn specimens were photograph in a mini studio to obtain maximum image quality and the distance between the camera and specimens when photograph was approximately $20 \mathrm{~cm}$. Photographs of the chelae and carapace of Macrobrachium are used for landmark configuration and analysis of shape variations.

The image of the Macrobrachium carapace and chelae section was analyzed using several software. Each chelae and carapace image digitized by using the tpsDig2 program to get the coordinates of the anatomical points on the chelae and carapace. Description of the prawn chelae section is done by using 6 landmark points and on the carapace part of the prawn using 10 landmark points. Images of each carapace and chelae of Macrobrachium were digitized 3 times to reduce digitization errors.

Geometric morphometrics analysis. The average value of landmark coordinates or consensus of each repeat is calculated with tpsrelw software (Rohlf, 2016b). Visualization of the results of digitizing species average with tpsSuper software. The visualization grid deformation shape of carapace and chelae each Macrobrachium species using tpsSplin software to see the variation of each point in the grid deformation is not uniform (Rohlf, 2004). Non-uniform components to show specific changes in certain anatomical points so that changes in the grid deformation can be known (Bookstein 1991; Zelditch et al., 2004).

Geometric Morphometrics and statistical analysis were carried out separately with the MorphoJ program (Klingenberg, 2011). Landmark anatomical points are optimally aligned with progress analysis to remove scale effects (Rohlf and Slice, 1990). Pattern variations in shape are assessed by performing Principal Component Analysis (PCA) in the Procrustes coordinates. PCA makes it possible to visualize and explore in the shape of variations by means of graphical representations of forms in all morphospace dimensions (Zelditch et al., 2012). PCA can be used to show the separation of individuals in a species (species) or between different species (interspecies) based on certain characters (Mann, 2007).

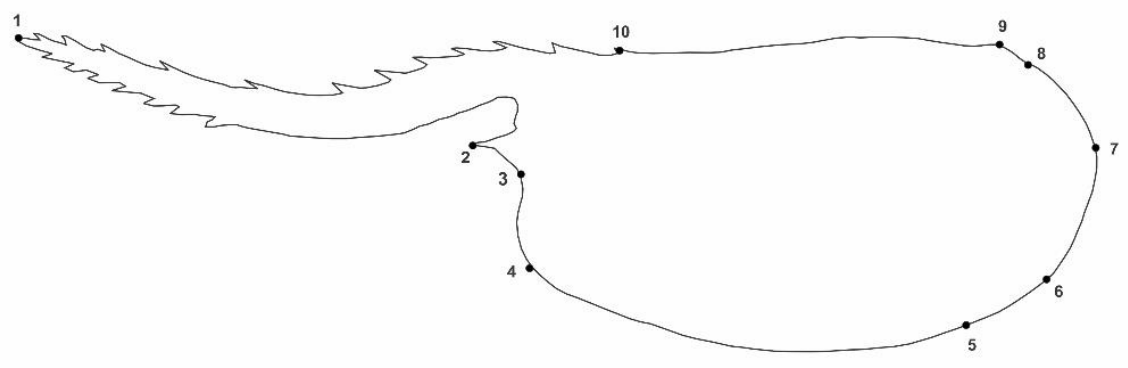

Figure 1. Position of anatomic or landmark points in the carapace of Macrobrachium section (Zimmermann et al., 2011). 1. tip of rostrum; 2. tip of ocular spines; 3 . ventral ocular spines; 4. front curvature of the bottom carapace; 5. ventral behind carapace; 6 . curve of back of carapace; 7. end of carapace; 8 . carapace curve back; 9 . upper carapace curve; 10. rostrum base spines 


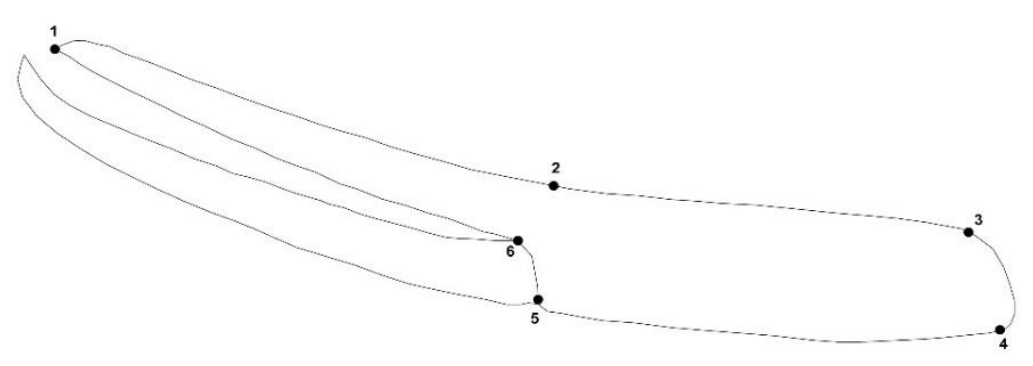

Figure 2. The position of anatomic or landmark points in the chelae of Macrobrachium section (Claverie \& Smith, 2010 with modifications). 1. Pollex tip; 2. Intersection between pollex and manus at the inferior boundary of propodus; 3. upper attachment point of carpus with propodus; 4. Lower attachment point of carpus with propodus; 5. Lower point that marks dactylus junction with propodus; 6 . The upper point that marks the dactylus junction with the propodus

Analysis of clustering defined with UPGMA method using PAST software based on Euclidean distance from the average form of each prawn population of each species to determine the level of similarity in each part of the carapace and chelae members of the genus Macrobrachium.

\section{RESULT AND DISCUSSION}

Grid Deformation. The results of the carapace shape grid deformation, $M$. rosenbergii have the highest bending energy value, which is equal to 0.083 and the most different carapace shapes, namely at point 1 , which shows a longer rostrum. Overall differences in landmark points are located at points $1,2,4$, and 10 which show variations in the shape of rostrum on each of the carapace of Macrobrachium.

The results of the chelae shape grid deformation (Figure 4), M. pilimanus have the highest bending energy value which is equal to 0.065 . The most distinct shape of $M$. pilimanus chelae is at landmark points 1,5 , and 6 which shows the shape of the pollex and the narrowed and short propodus M. lar has the smallest bending energy value which is equal to 0.008 .
Principal Component Analysis (PCA). Distribution of Macrobrachium in morphospace (Figure 5). Based on carapace shape, it shows that the population of $M$. rosenbergii has the most different carapace shape and independent because of the longest shape of rostrum among other prawns (Figure 3). PCA shows a large dispersion across morphospace between species groups (Figures 5). The first two PCs (Principal Components) explained $82.66 \%$ of the total variation in carapace shape with PC 1 explaining $75.11 \%$, PC 2 explained $7.55 \%$ of the total variation.

The distribution of Macrobrachium in morphospace (Figure 6) based on variations in the shape of the chelae shows that the population of Macrobrachium has a high level of overlap, except for the population of $M$. pilimanus which is different in shape from the others. M. pilimanus has a short pollex shape and a narrowed propodus compared to other prawn chelae (see Figure 4). Macrobrachium chelae shape variation based on PCA analysis shows the first two PCs (Principal Component) which explained $87.56 \%$ of the total variation of the chelae shape with PC 1 explaining $55.49 \%$ and PC 2 explaining $32.07 \%$ of the total variation. 


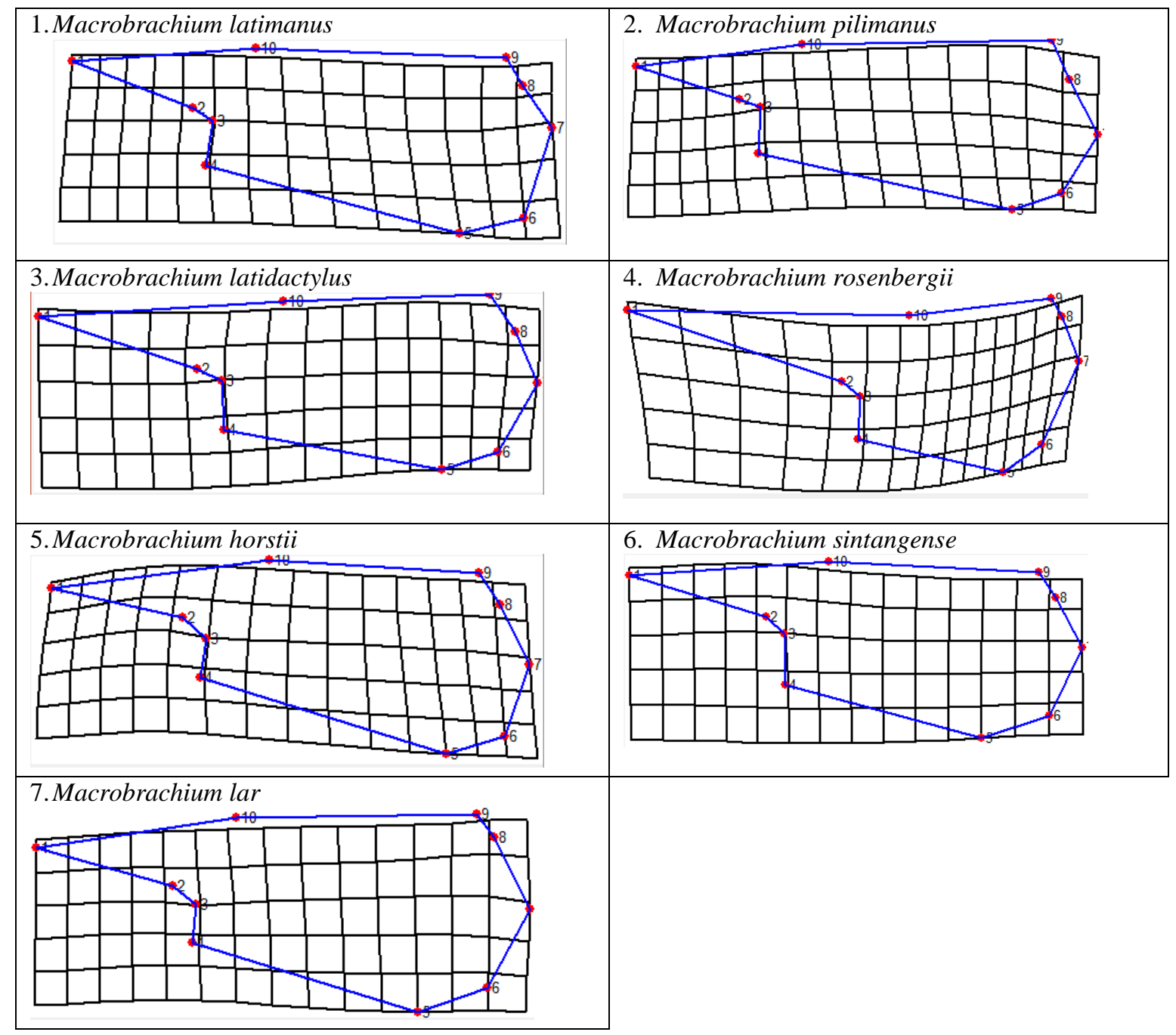

Figure 3. Variation in shape in the grid deformation of each carapace Macrobrachium

Visualization of differences in shape and changes in shape is the main objective and shows variations in morphometric geometry. The grid deformation functions to obtain visualization of shape variations based on the location of each landmark point between Macrobrachium species. Bookstein (1991) proposed the use of the Thin Plate Spline (TPS) function to fit the differences in landmark position in one organism relative to its position elsewhere. The shape of each grid deformation is influenced by bending energy, which is a measure of the difference in shape between two landmark configurations that do not require superimposition of procrustes from configuration and are usually not used for statistical analysis (Mitteroecker and Gunz, 2009).

Grid deformation (Figures 3 and 4) shows the direction of movement of landmark points in the carapace and chelae Macrobrachium. Based on the results of the carapace shape grid deformation, $M$. rosenbergii has the greatest bending energy value, which is equal to 0.08283 and the most different carapace forms, namely at point 1 , which shows a longer rostrum. It can be seen in the TPS grid deformation, M. Rosenbergii which has a box that extends to the left lateral direction at landmark points 1 and 2 while at the landmark points $4,5,6,7,8,9$, and 10 more narrow to the right lateral. M. Pilimanus has the second largest bending energy value of 0.05505 . Whereas the other 5 Macrobrachium species have different shapes which are not much different from each other and can be known from the energy bending magnitude of each species. $M$. sintangense has the lowest bending energy value of 0.014 so that the shape of the carapace based on the grid deformation has a 
shape that is close to the average shape because it only requires a small bending energy to change the shape to average shape. Overall differences in landmark points are located at points $1,2,4$, and 10 which show variations in the form of rostrum on each of the carapace of Macrobrachium.

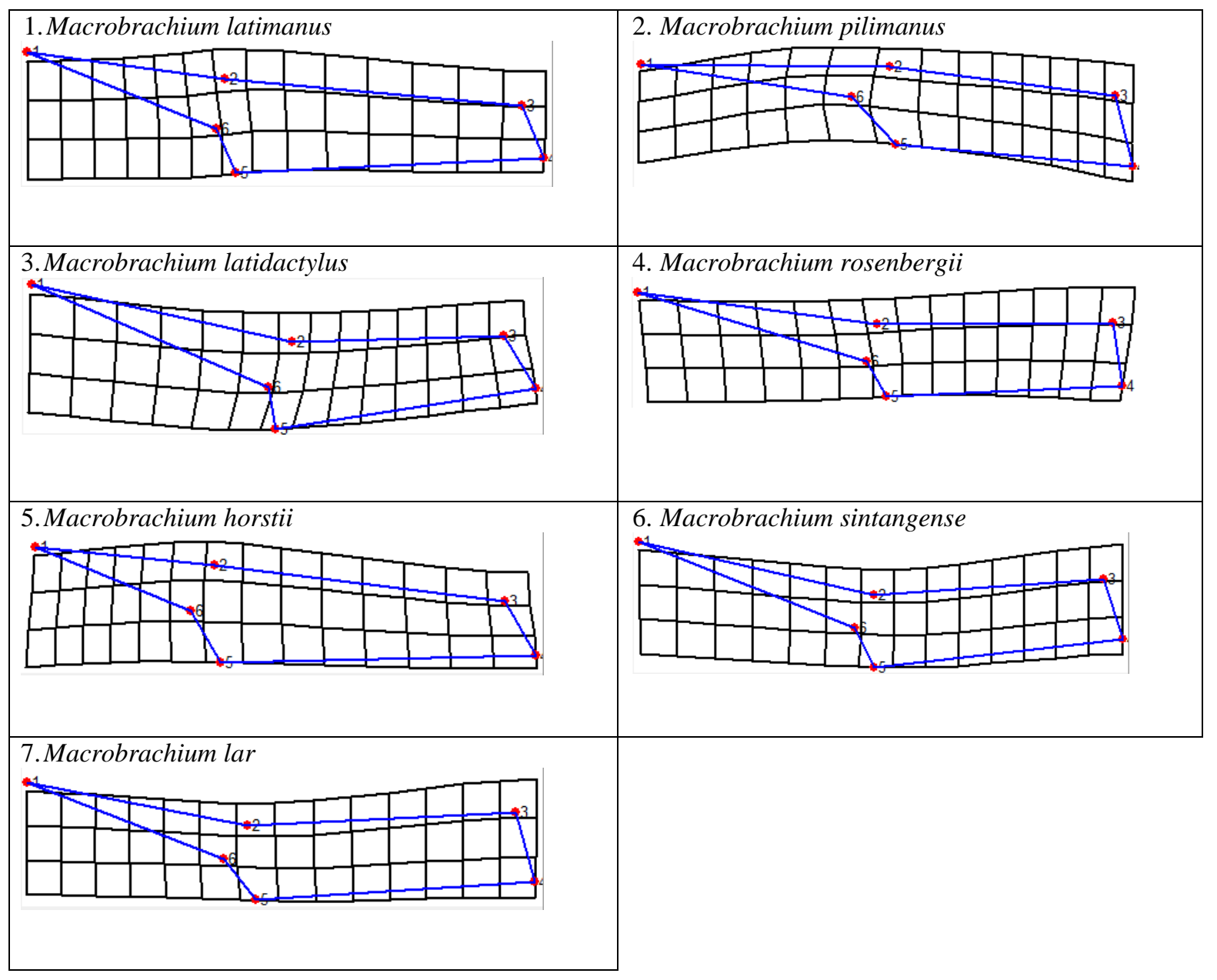

Figure 4. Variation in shape in the grid deformation of each chelae Macrobrachium

Based on the results of the chelae shape grid deformation (Figure 4), M. pilimanus prawn has the greatest bending energy value, which is equal to 0.065 which indicates the amount of energy required by the shape of the chelae to change greater than the shape of the chelae of the other species most different at landmarks 1,5 , and 6 which show a narrow and short form of pollex and propodus and can be seen on the $M$. pilimanus grid deformation landmark points 5 and 6 that move to the right lateral so that the pollex looks shorter than the chelae other prawn species. $M$. lar has the smallest bending energy value that is equal to 0.00819 , meaning that the shape of the M. lar requires only a small amount of energy to resemble the shape of the average chelae. 


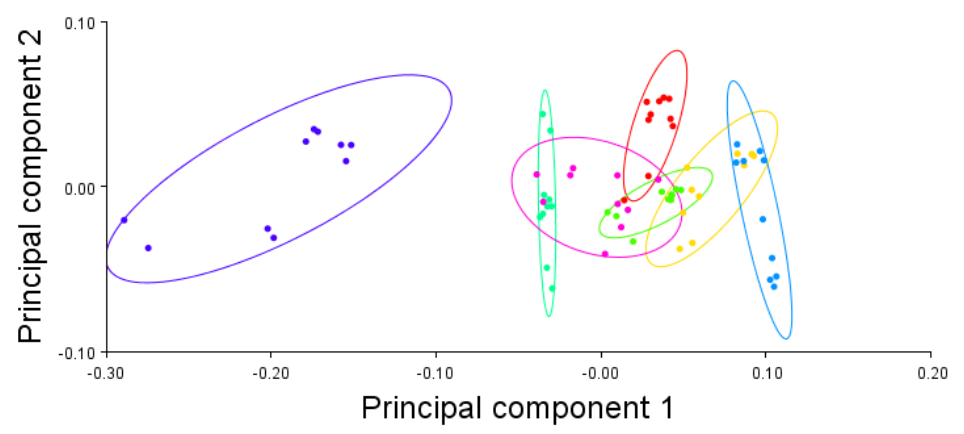

Figure 5. PCA variations in the shape of the carapace of Macrobrachium. $\mathrm{PC} 1=75.11 \%, \mathrm{PC} 2=7.55 \%$, total variation of $82.66 \%$. hor: M. horstii, lam: M. latimanus, lar: M. lar, lat: M. latidactylus, pills: M. pilimanus, ros: $M$. rosenbergii, sin: $M$. sintangense

PCA visualizes variations in carapace shape on each PC axis. PC 1 describes variations in the size and shape of rostrum. The specimen with the highest positive score on PC 1, M. pilimanus, has short rostrum, the tip of the ocular and ventral thorns of the ocular spines which approach the rostrum, the lower front carapace grooves, and the high rostrum spines. The specimen with the highest negative score on PC 1 is $M$. rosenbergii, has long rostrum, the tip of the ocular and ventral spines ocular spines away from the rostrum, short lower carapace front grooves, and low rostrum base spines. In Figure 5 shows the variation in shape on PC 1 , namely the $\mathrm{X}$ axis, where the $\mathrm{x}$ axis separates morphospace into two parts, the right positive score and the negative score on the left. While overlapping specimens in the middle of the morphospace show variations in the shape of the carapace that are not much different. PC 2 describes variations in the size and shape of the carapace width. In Figure 5 shows the variation in shape on PC 2, the $\mathrm{Y}$ axis, where the $\mathrm{Y}$ axis separates morphospace into two sides, the upper side with a positive score and the lower side shows a negative score. Based on PCA morphospace, PC 2 only occupies $7.55 \%$ of variation, which means that the variations in the shape on the $\mathrm{Y}$ axis do not affect all species and on the $\mathrm{Y}$ axis all species overlap.

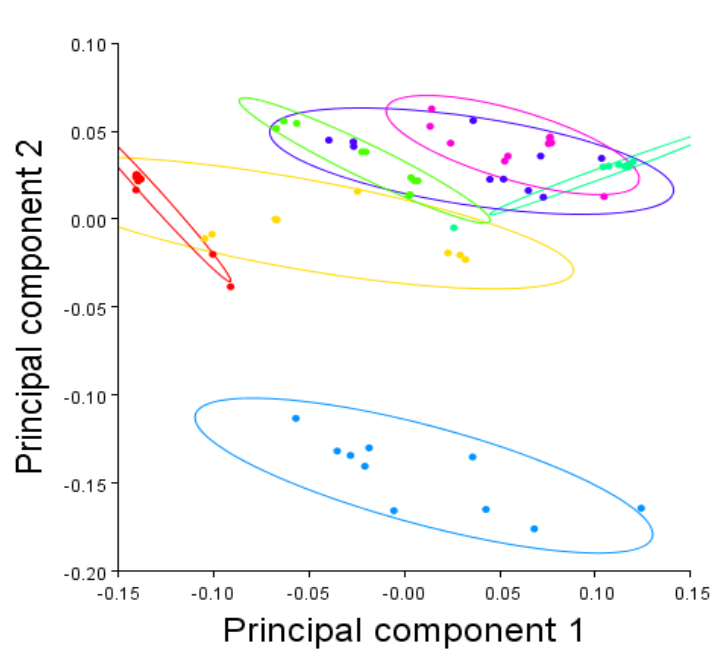

Figure 6. PCA (Principal Component Analysis) from the variation of Macrobrachium chelae shape. PC1 $=55.493 \%$, $\mathrm{PC} 2=32.072 \%$, total variation of $87.565 \%$. hor: M. horstii, lam: M. latimanus, lar: M. lar, lat: M. latidactylus, pil: . pilimanus, ros: $M$. rosenbergii, sin: M. sintangense 
PC 1 and PC 2 (Figure 6) describe variations in the size of the form of pollex and propodus. PC 1 shows that all species overlap on the $\mathrm{X}$ axis so that variations in the shape of the chelae spread by the $X$ axis from the most positive scores that have the form of extreme claws up to the most negative scores, together have a form that almost resembles the average form of a species namely pollex width and length and a wide but shorter propodus. Each species in PCA analysis does not stand alone but in the form of a variable data collection that is combined and produces new variable forms to see variations in the overall shape. On PC 2 it can be seen from the distribution on the $\mathrm{Y}$ axis, the specimen with the highest negative score on PC 2, namely M. pilimanus has a small pollex and a small propodus. While other specimens in positive scores overlap which shows the shape between them similar. $M$. horstii only overlaps with $M$. latimanus so that the shape of chelae $M$. horstii is more similar to M. latimanus.
Clustering Analysis. The dendogram shows the population grouping of Macrobrachium based on the similarity of carapace and chelae. The first group includes $M$. latidactylus and $M$. sintangense, the second group includes $M$. horstii and M. latimanus. $M$. lar is the only species that shows the similarity of the shape of the carapace and chelae with the two groups. $M$. rosenbergii is on a different line which shows the different shape of the carapace, which is the striking rostrum which is longer and curves upwards. However, in the shape of $M$. rosenbergii chelae it has a form similar to $M$. latidactylus and $M$. sintangense. $M$. pilimanus occupies the most different position and separates from all populations of Macrobrachium which shows differences in the shape of the carapace, namely the short shape of rostrum and the high base of rostrum spines and narrowed shape of the chelae with pollex and propodus.

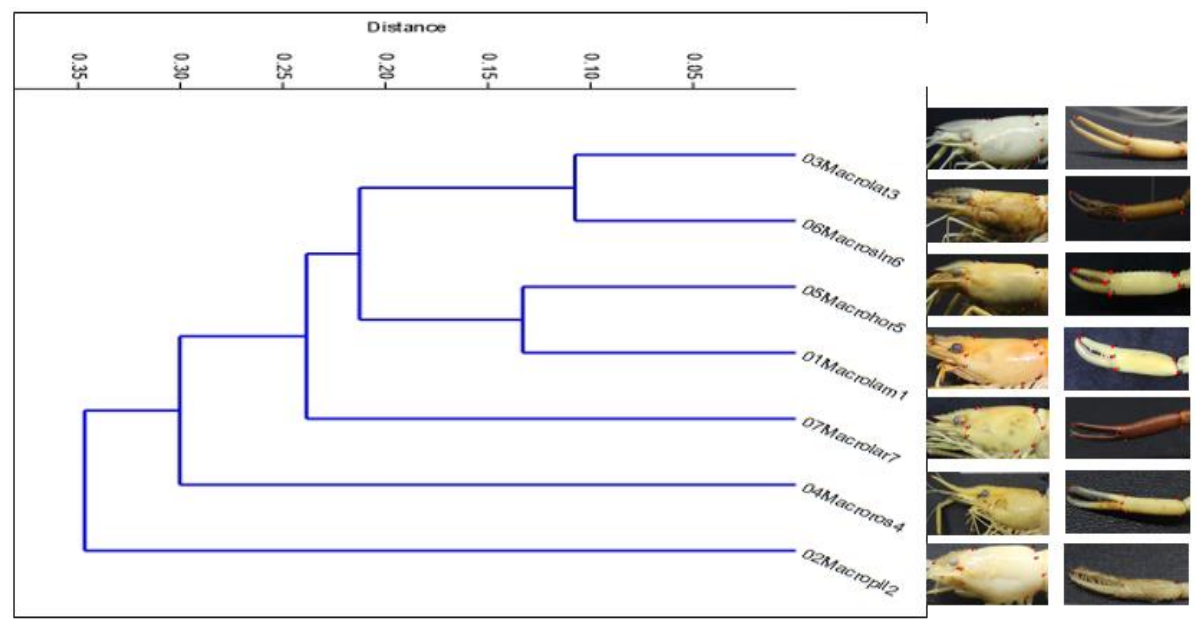

Figure 7. Dendogram with UPGMA method on the carapace and chelae parts based on similarity using the Euclidean Distance matrix

The resulting dendogram (Figure 7) shows a grouping of populations of Macrobrachium based on similarities in the shape of the carapace and chelae. The first group includes $M$. latidactylus and $M$. sintangense which are a group of prawn that have a carapace shape with a straight forward rostrum and a chelae shape with a long pollex and a narrowed propodus. The two species clustered with a similarity distance of $89 \%$ and the difference between $M$. latidactylus and $M$. sintangense was the shape of the back end of the carapace and the curved shape of the pollex on the chelae. $M$. latidactylus has the shape of the back end of the carapace which is not protruding and the shape of the pollex tip is long and not curved and the propodus has a proportion that is almost the same as pollex, whereas in $M$. sintangense it has 
a prominent carapace end and pollex tip curved and the proportion of the length of the propodus is longer than the pollex. The second group includes $M$. horstii and M. latimanus which are a group of prawn that have a carapace shape with short rostrum and a chelae shape with small pollex and a wide propodus. The two species clustered with a similarity distance of $87 \%$ and the difference between $M$. horstii and $M$. latimanus was the form of the rostrum and the shape of the pollex on the chelae. M. horstii has a thin form of rostrum and a short form of pollex and a straight forward pollex, whereas in M. latimanus it has the form of a rostrum which extends towards the ventral and shape a short pollex and curved pollex tip. M. lar is the only prawn species that shows the similarity of the shape of the carapace and chelae with the two groups, where the carapace form in M. lar is more similar to the second group while the shape of the chelae is more similar to the first group. $M$. rosenbergii is on a different line which shows the different shape of the carapace, which is the striking rostrum which is longer and curves upwards. However, in the shape of chelae $M$. rosenbergii it has a shape like $M$. latidactylus and $M$. sintangense. $M$. pilimanus occupies the most different position and separates from all populations of Macrobrachium which shows differences in the shape of the carapace, namely the short form of rostrum and the high base of rostrum spines and narrowed shape of the clit with pollex and propodus.

The resulting dendogram displays the grouping of Macrobrachium species based on the similarity of the shape of the carapace and chelae and does not indicate a grouping based on genetic kinship. The shape of the carapace and chelae in each Macrobrachium species have not been consistent in terms of grouping. This can occur because the basis of grouping is a variation of morphological shape that is influenced by behavior and environmental factors or different habitats in each species of Macrobrachium.

\section{CONCLUSION}

Variation of the Macrobrachium carapace shape represented by the length of the rostrum and the shape of the carapace. $M$. rosenbergii has the most different form of carapace, which is a long rostrum and a carapace that is not wide with low rostrum spines. M. pilimanus has a short shape of rostrum and wide carapace with high rostrum base spines. M. latimanus, $M$. horstii, and $M$. lar have rostrum length and carapace shape which are more similar to $M$. pilimanus, whereas $M$. sintangense and $M$. latidactylus have rostrum length and carapace shape between $M$. rosenbergii and $M$. pilimanus. In the prawn chelae section, members of the genus Macrobrachium have variations in shape represented by the shape of the pollex and the width of the propodus. $M$. pilimanus has the most distinct shape of chelae, which is a narrow shape of pollex and a narrowed propodus. $M$. rosenbergii, $M$. lar, $M$. sintangense, and $M$. latidactylus have nearly the same shape of claws, namely the form of pollex and the long and narrow shape of the propodus. $M$. latimanus and $M$. horstii have almost the same shape of chelae, namely the shape of pollex and propodus which are wide and short when seen morphologically directly, the claws of $M$. latimanus and $M$. horstii look more sturdy.

\section{REFERENCES}

Adam DC, Rohlf FJ, Slice DE. 2004. Geometric morphometrics: Ten years of progress following the 'revolution. Italian Journal of Zoology. vol 71(1): 5-16. doi: https://doi.org/10.1080/112500004093565 45.

Adams DC, Rohlf FJ and Slice DE. 2013. A field comes of age: Geometric morphometrics in the 21st century. Hystrix. vol 24 (1): 7-14. doi: https://doi.org/10.4404/hystrix-24.1-6283.

Bookstein FL. 1991. Morphometric Tools for Landmark Data: Geometry and Biology. New York: Cambridge University Press. (xvii): 435.4 doi: https://doi.org/10.1017/CBO97805115730 64.

Claverie T and Smith I. 2010. Allometry and sexual dimorphism in the chela shape in the squat lobster Munida rugosa. Aquatic 
Biology. vol 8: 179-187. doi: https://doi.org/10.3354/ab00233.

Klingenberg CP. 2011. MorphoJ: An integrated software package for geometric morphometrics. Molecular Ecology Resources. vol 11(2): 353-357. doi: $\quad$ https://doi.org/10.1111/j.17550998.2010.02924.x.

Klingenberg CP. 2013. Visualizations in geometric morphometrics: How to read and how to make graphs showing shape changes. Associazione Teriologica Italiana. Hystrix, the Italian Journal of Mammalogy. vol 24(1): 15-24. doi: https://doi.org/10.4404/hystrix-24.1-7691.

Mann AM. 2007. A Taxonomic Investigation of the Black Ratsnake, Elaphe o. obsoleta (Say) [Reptilia, Squamata, Colubridae], in West Virginia using Morphometric Analyses. [Thesis]. Huntington: The Graduate College of Marshall University.

Mitteroecker P and Gunz P. 2009. Advances in Geometric morphometrics. Evolutionary Biology. vol 36 (2): 235-247. doi: https://doi.org/10.1007/s11692-009-9055$\mathrm{x}$.

New MB. 2002. Farming Freshwater Prawn. A manual for the culture of the giant river prawn (Macrobrachium rosenbergii). FAO Fisheries Technical Paper (428). Rome. p. 1-3.

Rohlf FJ and Slice DE. 1990. Extensions of the Procrustes Method for the Optimal Superimposition of Landmark. Systematic Zoology. vol 39 (1): 40-59. doi: https://doi.org/10.2307/2992207.

Rohlf FJ. 2004. tpsSplin Version 1.20. Stony Brook (US): New York: Department of Ecology and Evolution-State University.
Rohlf FJ. 2016. tpsRelw Version 1.53. Stony Brook (US): New York: Department of Ecology and Evolution-State University.

Rufino M, Abelló P, Yule AB. 2009. Male and female carapace shape differences in Liocarcinus depurator (Decapoda, Brachyura): An application of geometric morphometric analysis to crustaceans. Italian Journal of Zoology. vol 71(1): 7983. doi: https://doi.org/10.1080/112500004093565 54.

Valencia DM and Campos MR. 2007. Freshwater prawns of the genus Macrobrachium Bate, 1868 (Crustacea: Decapoda: Palaemonidae) of Colombia. Magnolia Press. Zootaxa. vol 1456: 1-44. doi: http://dx.doi.org/10.11646/zootaxa.1456.1

Zelditch ML, Swiderski DL, Sheets HD, and Fink WL. 2004. Geometric morphometrics for biologists: A primer. Elsevier Academic Press San Diego. vol. 95, p. 443. doi: https://doi.org/10.1016/B978-0-12386903-6.00001-0.

Zelditch ML, Swiderski DL, and Sheets HD. 2012. Geometric morphometrics for biologists: A primer second edition. Geometric Morphometrics for Biologists. pp. $1-475$. doi: https://doi.org/10.1016/B978-0-12386903-6.00001-0.

Zimmermann G, Bosc P, Valade P, Cornette R, Ame 'ziane N, and Debat V. 2011. Geometric morphometrics of carapace of Macrobrachium australe (Crustacea: Palaemonidae) from Reunion Island. Acta Zoologica. vol 93 (4): 492-500. doi: https://doi.org/10.1111/j.1463 6395.2011.00524.x. 